\title{
Effect of Reynolds Number on Aerodynamics of Airfoil with Gurney Flap
}

\author{
Shubham Jain, ${ }^{1}$ Nekkanti Sitaram, ${ }^{2}$ and Sriram Krishnaswamy ${ }^{3}$ \\ ${ }^{1}$ Department of Aerospace Engineering, PEC University of Technology, Sector 12, Chandigarh 160012, India \\ ${ }^{2}$ Department of Mechanical Engineering, IIT Madras, Chennai, Tamil Nadu 600036, India \\ ${ }^{3}$ Department of Mechanical Engineering, BITS Pilani, Hyderabad Campus, Jawahar Nagar, \\ Shameerpet Mandal, Hyderabad, Telangana 500078, India
}

Correspondence should be addressed to Shubham Jain; shubhamjain.jld@gmail.com

Received 12 June 2015; Revised 23 August 2015; Accepted 26 August 2015

Academic Editor: Jechin Han

Copyright (C) 2015 Shubham Jain et al. This is an open access article distributed under the Creative Commons Attribution License, which permits unrestricted use, distribution, and reproduction in any medium, provided the original work is properly cited.

\begin{abstract}
Steady state, two-dimensional computational investigations performed on NACA 0012 airfoil to analyze the effect of variation in Reynolds number on the aerodynamics of the airfoil without and with a Gurney flap of height of 3\% chord are presented in this paper. RANS based one-equation Spalart-Allmaras model is used for the computations. Both lift and drag coefficients increase with Gurney flap compared to those without Gurney flap at all Reynolds numbers at all angles of attack. The zero lift angle of attack seems to become more negative as Reynolds number increases due to effective increase of the airfoil camber. However the stall angle of attack decreased by $2^{\circ}$ for the airfoil with Gurney flap. Lift coefficient decreases rapidly and drag coefficient increases rapidly when Reynolds number is decreased below critical range. This occurs due to change in flow pattern near Gurney flap at low Reynolds numbers.
\end{abstract}

\section{Introduction}

A Gurney flap (GF) is a microtab fitted perpendicular to the airfoil near the trailing edge on its pressure surface which increases the lift by altering the Kutta condition and increasing effective camber. They are extensively used on helicopter stabilizers [1]. Considerable efforts are carried out to study the effects of Gurney flap on the airfoil aerodynamics [2-4]. Many investigations are undertaken to determine the effect of various parameters of Gurney flap such as height, position, mounting angle, and configurations. But most of the investigations are performed at high Reynolds numbers above critical range. Mueller and Batill [5], Selig et al. [6], Brown and Filippone [7], and Traub and Agarwal [8] have studied the performance of airfoils at low Reynolds numbers but a systematic investigation is still not available. For smaller UAVs and MAVs, airfoil chord and velocity of aircraft are lower; hence, they operate at low Reynolds numbers. Reynolds numbers between $\operatorname{Re}=3 \times 10^{4}$ and $7 \times 10^{4}$ are of great interest to MAV designers [9]. Most UAVs operate at $\mathrm{Re}=5 \times 10^{4}$ and $3 \times 10^{5}$ [10]. Gurney flaps are also used in turbomachinery to improve their performance at low Reynolds number due to their inexpensive method of lift enhancement [11].

A semiempirical formula linking flap height to free stream velocity and the airfoil chord is proposed by Brown and Filippone [7]:

$$
h_{\mathrm{opt}}=37.155\left(\frac{\mathrm{Ch}^{0.8}}{U^{0.2}}\right) \text {, }
$$

where $h_{\text {opt }}$ is optimum GF height $(\mathrm{mm})$, Ch is chord length $(\mathrm{m})$, and $U$ is free stream velocity $(\mathrm{m} / \mathrm{s})$.

Keeping this in mind, computations on the effect of six Reynolds numbers from high Reynolds number to low Reynolds number well below the critical range are carried out to analyze the performance of the airfoil. 


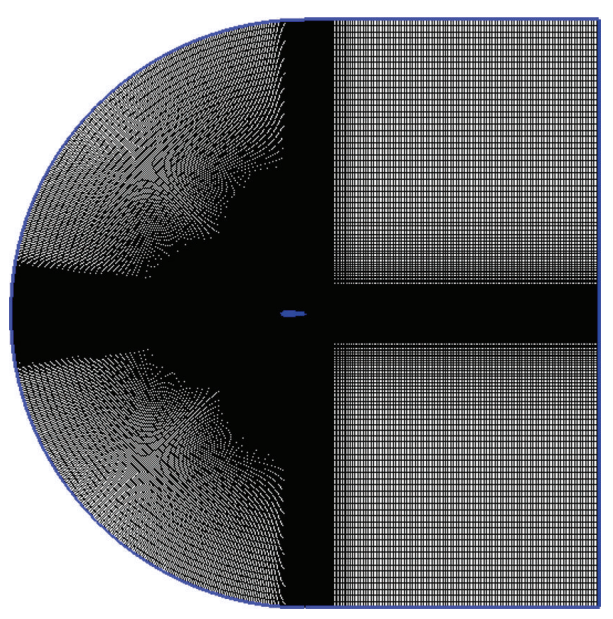

(a)
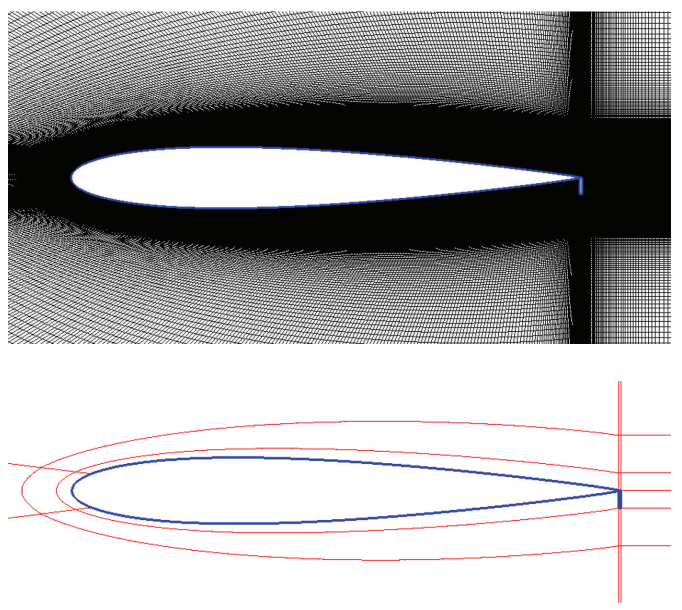

(b)
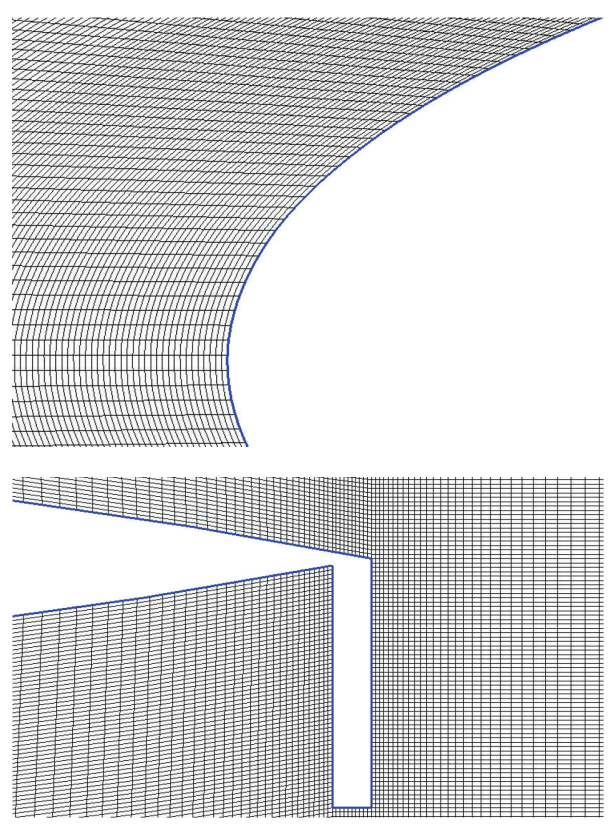

(c)

FIGURE 1: (a) Entire domain of the grid used. (b) Near surface grid and blocking near the airfoil surfaces. (c) Boundary layer elements near the leading and trailing edges of the airfoil.

\section{Computational Methodology}

2.1. Geometry and Grid Generation. The airfoil considered in this study is NACA 0012 airfoil with chord length of $5 \mathrm{~cm}$. C-type domain and grid are created in ICEM-CFD with farfield boundaries 12.5 chords away from trailing edge in all directions (Figure 1(a)). Grid is generated in ICEM-CFD with at least 300,000 nodes. Flow around the airfoil with GF is highly complex with high intensity vortices. Hence, first layer with cell width of $0.025 \mathrm{~mm}$ is generated up to $3 \%$ chord length around the airfoil. Second layer with cell width of $0.035 \mathrm{~mm}$ up to next $7 \%$ of the airfoil chord is generated around the airfoil as illustrated in the blocking strategy shown in Figure 1(b). Thereafter cell growth ratio of 1.05 is set up to far-field. Wall $y^{+}$values along the airfoil surface are kept below 1 to account for low Reynolds number flow regime. Boundary layer elements near the leading and trailing edges of the airfoil are shown in Figure 1(c).

2.2. Turbulence Modeling. Previous studies show that out of two equation RANS based models of FLUENT, $\kappa-\varepsilon$ RNG model is best suited for the present airfoil study $[12,13]$. However these computations are carried out at a higher Reynolds number of $2.1 \times 10^{6}$. RNG theory accounts for low Reynolds number effects. However effective use of this feature depends on appropriate treatment [14]. Hence it was decided to explore one-equation Spalart-Allmaras (SA) turbulence model for the present computations at low Reynolds numbers and compare these results with those of $\kappa-\varepsilon$ RNG model for 
TABLE 1: Comparison of $C_{L}$ predicted by $\kappa-\varepsilon$ RNG and SA models at $\operatorname{Re}=2.1 \times 10^{6}$.

\begin{tabular}{ccccccc}
\hline$\alpha$ & RNG & SA & Expt. & \multicolumn{3}{c}{$\begin{array}{c}\text { difference between } \\
\text { RNG and Expt. SA and Expt. RNG and SA }\end{array}$} \\
\hline $0^{\circ}$ & 0.542 & 0.538 & 0.406 & 25.1 & 24.5 & 0.7 \\
$4^{\circ}$ & 0.990 & 0.997 & 0.868 & 12.3 & 12.9 & -0.7 \\
$8^{\circ}$ & 1.405 & 1.435 & 1.285 & 8.5 & 10.5 & -2.1 \\
$12^{\circ}$ & 1.756 & 1.827 & 1.646 & 6.3 & 9.9 & -4.0 \\
\hline
\end{tabular}

improved predictions. Validation is done on an airfoil with $1 \mathrm{~m}$ chord length.

2.3. Boundary Conditions and Solver Settings. The airfoil boundary is assigned as solid-wall with no-slip condition while inlet is assigned as velocity inlet and outlet is assigned as pressure-outlet conditions. Density based implicit solving scheme is used with the flow medium being air and Mach number less than 0.3. Hence the fluid is assumed to be incompressible with constant density of $1.225 \mathrm{~kg} / \mathrm{m}^{3}$ and dynamic viscosity of $1.7894 \times 10^{-5} \mathrm{~kg} / \mathrm{m}$-s. Under relaxation factors for all the transport variables are set to 0.8 . Solution initialization is computed from velocity inlet followed by FMG initialization with solution steering. Equations are solved until a convergence criterion of $10^{-5}$ for all the residuals is satisfied.

\section{Results and Discussions}

3.1. Validation. Lift and drag coefficients, $C_{L}$ and $C_{D}$, for the airfoil of $1 \mathrm{~m}$ chord are computed with $\kappa-\varepsilon$ RNG and SA turbulence models and compared at high Reynolds numbers $\left(2.1\right.$ and $\left.3.0 \times 10^{6}\right)$ and at a lower Reynolds number $(5.0 \times$ $10^{5}$ ). The results are presented as a function of angle of attack in Figure 2. Comparison is also made with available experimental results at $\operatorname{Re}=2.1 \times 10^{6}[3]$.

For high Reynolds number, $C_{L}$ values obtained by both models are very close (Table 1). Masoud et al. [15] also found good agreement with experimental values of lift and drag coefficients for NACA 23018 airfoil using Spalart-Allmaras (SA) turbulence model. At the lower Reynolds number, the $C_{L}$ values predicted by $\kappa-\varepsilon$ RNG model decrease rapidly more than SA model. This difference also increases at higher angles of attack as shown in Figure 2. Similar trends are noticed for drag coefficient. Both computational models predict higher drag than experimental results. However the $C_{D}$ values predicted by the SA model are close to the experimental values (Table 2) and almost $40 \%-50 \%$ lower than $\kappa-\varepsilon$ RNG model at $\operatorname{Re}=5.0 \times 10^{5}$. Based on the validation studies, it was decided to use SA model for all computations at low Reynolds numbers. It is quite possible that improved predictions particularly drag coefficient may be obtained using advanced turbulence models. This is demonstrated by Arko and McQuilling [16], who used advanced turbulence models of Abe et al. [17, 18] and Kato and Launder [19]. However FLUENT does not have these turbulence models available.

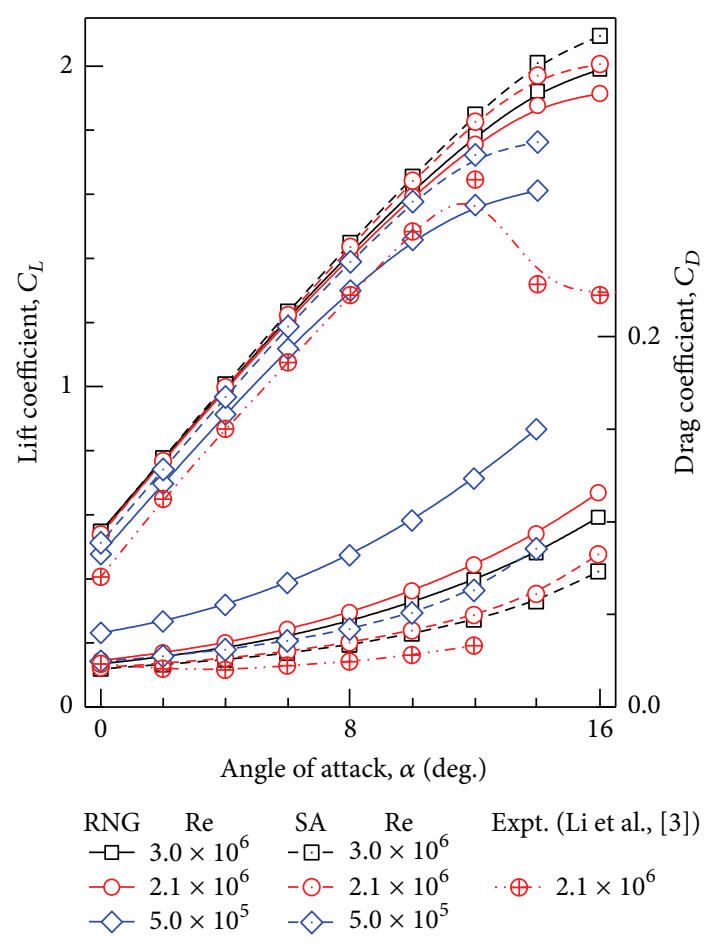

Figure 2: Variation of $C_{L}$ and $C_{D}$ with AoA for $\kappa-\varepsilon$ RNG and SA turbulence models.

3.2. Effect of Reynolds Number on Aerodynamics of the Airfoil without and with Gurney Flap. Computations are carried out for the airfoil without and with a GF of height of $3 \%$ chord at six different Reynolds numbers $\left(\mathrm{Re}=3.0 \times 10^{5}, 1.5 \times 10^{5}, 1.0 \times\right.$ $10^{5}, 8 \times 10^{4}, 5 \times 10^{4}$ and $\left.3 \times 10^{4}\right)$. Based on the equation for optimum GF given by Traub and Agarwal [8], the optimum GF height varies from 2.7 to $4.2 \%$ of airfoil chord as Reynolds number decreases from $3.0 \times 10^{5}$ to $3.0 \times 10^{4}$. Hence a GF of height of $3 \%$ of airfoil chord is chosen for computations at all Reynolds numbers. At low Reynolds numbers, SA turbulence model works better than $\kappa-\varepsilon$ RNG turbulence model. Hence the former model is used for all the following investigations.

3.2.1. Lift Coefficient. The lift coefficient $C_{L}$ versus AoA characteristics for the airfoil without and with Gurney flap is presented in Figure 3. At all Reynolds numbers, the lift coefficient for the airfoil with GF is always higher than the lift coefficient for the airfoil without GF at all angles of attack below the stall angle. The lift coefficient obtained at AoA = $8^{\circ}$ for the airfoil with GF is higher than maximum lift coefficient obtained for the airfoil without GF. As Reynolds number decreases, the lift coefficient also decreases for both cases: without and with GF. The extent to which Reynolds number affects the performance varies for both the cases and is presented in Table 3 . The zero lift angle seems to become more negative as Reynolds number increases (it is estimated to be -2 to $-3^{\circ}$ as Reynolds number increases from $3.0 \times 10^{4}$ to $\left.3.0 \times 10^{5}\right)$. This suggests that the effect of GF is to increase the effective camber of the airfoil. However the effective camber decreases as Reynolds number decreases. 
TABLE 2: Comparison of $C_{D}$ predicted by $\kappa-\varepsilon$ RNG and SA models at $\operatorname{Re}=2.1 \times 10^{6}$.

\begin{tabular}{|c|c|c|c|c|c|c|}
\hline \multirow{2}{*}{$\alpha$} & \multirow{2}{*}{ RNG } & \multirow{2}{*}{ SA } & \multirow{2}{*}{ Expt. } & \multicolumn{3}{|c|}{$\%$ difference between } \\
\hline & & & & RNG and Expt. & SA and Expt. & RNG and SA \\
\hline $0^{\circ}$ & 0.0250 & 0.0212 & 0.0233 & 6.8 & -9.9 & 15.2 \\
\hline $4^{\circ}$ & 0.0347 & 0.0264 & 0.0199 & 42.7 & 24.6 & 23.9 \\
\hline $8^{\circ}$ & 0.0510 & 0.0348 & 0.0245 & 52.0 & 29.6 & 31.8 \\
\hline $12^{\circ}$ & 0.0766 & 0.0496 & 0.0331 & 56.8 & 33.3 & 35.2 \\
\hline
\end{tabular}

TABle 3: Percentage Decrement in $C_{L}$ per every 10,000 decrease in Reynolds number for different ranges of Reynolds number.

\begin{tabular}{|c|c|c|c|c|c|}
\hline \multicolumn{6}{|c|}{ Reynolds number range } \\
\hline AoA, $\alpha$ & $\begin{array}{l}3.0 \times 10^{5} \\
\text { to } 1.5 \times 10^{5}\end{array}$ & $\begin{array}{l}1.5 \times 10^{5} \\
\text { to } 1.0 \times 10^{5}\end{array}$ & $\begin{array}{c}1.0 \times 10^{5} \\
\text { to } 8.0 \times 10^{4}\end{array}$ & $\begin{array}{c}8.0 \times 10^{4} \\
\text { to } 5.0 \times 10^{4}\end{array}$ & $\begin{array}{c}5.0 \times 10^{4} \\
\text { to } 3.0 \times 10^{4}\end{array}$ \\
\hline \multicolumn{6}{|l|}{$4^{\circ}$} \\
\hline Without GF & 0.12 & 0.25 & 0.37 & 0.57 & 1.12 \\
\hline With GF & 0.18 & 0.49 & 0.86 & 1.57 & 3.41 \\
\hline \multicolumn{6}{|l|}{$8^{\circ}$} \\
\hline Without GF & 0.15 & 0.31 & 0.45 & 0.72 & 1.30 \\
\hline With GF & 0.20 & 0.50 & 0.82 & 1.40 & 2.74 \\
\hline \multicolumn{6}{|l|}{$12^{\circ}$} \\
\hline Without GF & 0.25 & 0.50 & 0.66 & 0.98 & 1.62 \\
\hline With GF & 0.42 & 0.85 & 1.17 & 1.65 & 2.51 \\
\hline
\end{tabular}

For the airfoil without GF, variation in $C_{L}$ values with Reynolds number is negligible at low angles of attack and increases slightly with increasing angle of attack. When Reynolds number is changed from $1.5 \times 10^{5}$ to $1.0 \times 10^{5}$, $\mathrm{C}_{L}$ decrement for $\mathrm{AoA}=4^{\circ}, 8^{\circ}$ and $12^{\circ}$ is $1.2 \%, 1.6 \%$, and $2.5 \%$, respectively. However, for the airfoil with Gurney flap, decrement in $C_{L}$ is almost the same at lower and intermediate angles of attack. The lift coefficient decreases rapidly near stall. Changing the Reynolds number from $1.5 \times 10^{5}$ to $1.0 \times$ $10^{5}$, decrement in $C_{L}$ values the airfoil with $\mathrm{GF}$ at $\mathrm{AoA}=4^{\circ}$ and $\mathrm{AoA}=8^{\circ}$ is $2.5 \%$, whereas it at $\mathrm{AoA}=12^{\circ}$ is $4.2 \%$.

Table 3 represents the percentage decrement in $C_{L}$ values for every 10,000 decreases in Reynolds number for different ranges of Reynolds number for the airfoil without and with GF. For AoA $=12^{\circ}$, percentage decrease in $C_{L}$ for the airfoil without GF for every 10,000 decreases in Reynolds number near critical Reynolds number range $\left(\operatorname{Re}_{\mathrm{cr}} \approx 1.5 \times 10^{5}\right)$ is 0.25 . Below critical range the percentage decrement increases from 0.6 for the Reynolds number range of $1.0 \times 10^{5}$ to $8.0 \times$ $10^{4}$ to 1.62 at very low Reynolds number range of $5.0 \times 10^{4}$ to $3.0 \times 10^{4}$. For AoA $=12^{\circ}$, percentage decrease in $C_{L}$ for the airfoil with GF for every 10,000 decreases in Reynolds number near critical range is 0.42 . Below critical range the percentage decrement increases from 1.17 for the Reynolds number range of $1.0 \times 10^{5}$ to $8.0 \times 10^{4}$ to 2.51 at very low Reynolds number range of $5.0 \times 10^{4}$ to $3.0 \times 10^{4}$. Hence it may be concluded that Gurney flap has adverse effects on lift coefficient as Reynolds number is decreased. However the lift coefficient for the airfoil with GF is always higher than that for the airfoil without GF at all Reynolds number and at all angles of attack.

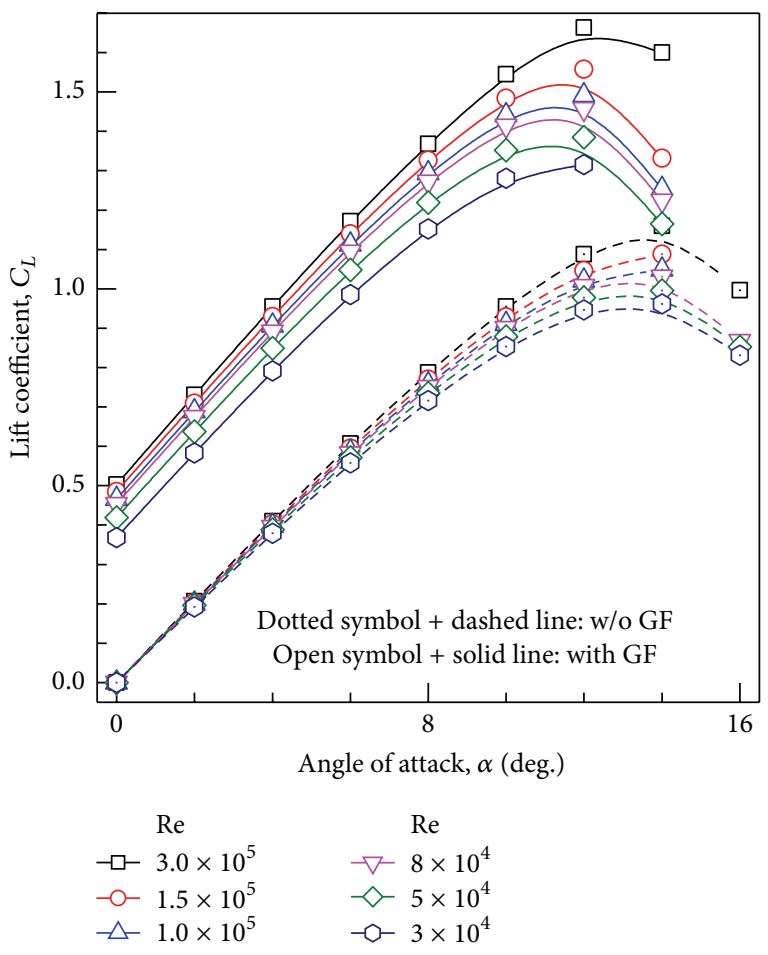

Figure 3: Variation of $C_{L}$ with AoA for different Reynolds numbers for the airfoil without and with GF.

The variation of the lift coefficient, $C_{L}$, as a function of Reynolds number at representative angles of attack is shown in Figure 4. This figure clearly shows the rapid decrease in $C_{L}$ 


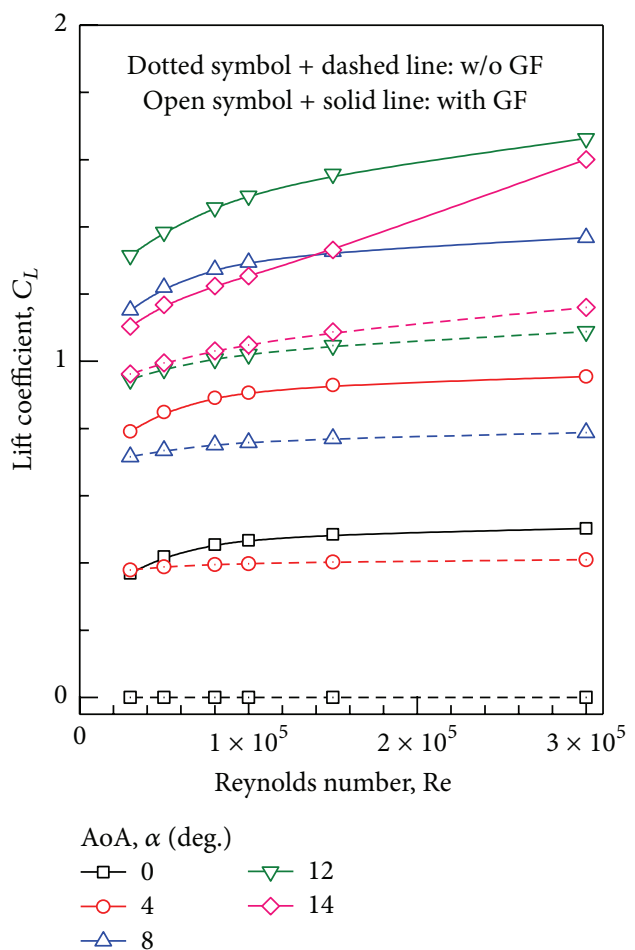

FIGURE 4: Variation of $C_{L}$ with Reynolds number for the airfoil without and with GF.

below the critical Reynolds number. This figure also shows the lift enhancement capability of Gurney flap at all Reynolds numbers. It is also noted that the performance of the airfoil without GF is less affected by variation in Reynolds number.

3.2.2. Drag Coefficients. Apart from decreasing $C_{L}$ with decreasing Reynolds number, $C_{D}$ is also found to increase as shown in Figure 5. For the sake of clarity, drag coefficient is presented at four Reynolds numbers only, namely, $3.0 \times$ $10^{5}, 1.5 \times 10^{5}, 8.0 \times 10^{4}$, and $3.0 \times 10^{4}$. Magnitude by which $C_{D}$ increases with decreasing Reynolds number is nearly independent of angle of attack. Increase in $C_{D}$ is very steep below critical Reynolds number as clearly shown in Figure 6. For higher Reynolds number range, the slope of $C_{D}$ increment is more for high AoA. Apart from lift enhancement capability of Gurney flap, increased drag penalty is also clearly visible. The $C_{D}$ obtained at $\mathrm{AoA}=12^{\circ}$ for the airfoil without GF is obtained at only AoA $=8^{\circ}$ with GF. However, the increase in $C_{D}$ is less for the airfoil with GF when compared to the airfoil without GF.

The percentage increment in $C_{D}$ values for every 10,000 decreases in Reynolds number for different ranges of Reynolds number for the airfoil without and with GF is presented in Table 4. For AoA $=12^{\circ}$, percentage increase in $C_{D}$ for the airfoil for every 10,000 decreases in Reynolds number near critical range is 1.39 , whereas below critical range the value increases from 3.19 for the Reynolds number range of $1.0 \times 10^{5}$ to $8.0 \times 10^{4}$ to 10.07 at very low Reynolds number range of $5 \times 10^{4}$ to $3 \times 10^{4}$. The respective values for the airfoil with GF are 1.3, 2.62, and 6.60 . Hence it may be

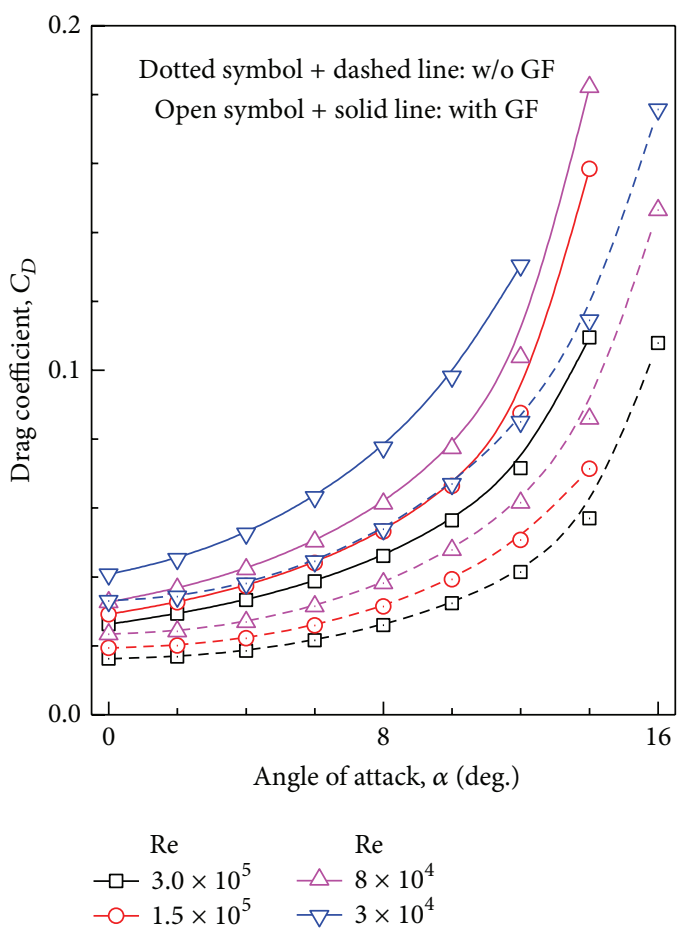

Figure 5: Variation of $C_{D}$ with AoA for different Reynolds numbers for the airfoil without and with GF.

concluded that Gurney flap has certain beneficial effects on drag coefficient as Reynolds number is decreased.

3.2.3. Lift-to-Drag Ratio. The lift-to-drag ratio is presented as $C_{L} / C_{D}$ at different angles of attack in Figure 7. From the figure it is evident that the lift-to-drag ratio for the airfoil with GF is higher than that for the airfoil without GF up to angle of attack of $8^{\circ}$. At this angle of attack, the lift-todrag ratios for the airfoil without and with GF are almost equal at all Reynolds numbers. Beyond this angle of attack, the lift-to-drag ratio for the airfoil with GF is lower than that for the airfoil without GF, indicating additional drag penalty due to GF. The difference between the lift-to-drag ratios for the airfoil without and with GF seems to be constant at all Reynolds numbers. However the difference between the liftto-drag ratios for the airfoil without and with GF at angle of attack of $14^{\circ}$ is substantially higher than that $12^{\circ}$. From Figure 3, it is evident that the airfoil with GF stalls at angle of attack of $12^{\circ}$ while the airfoil without GF stalls at angle of attack of $14^{\circ}$. This is the reason for the large difference at angle of attack of $14^{\circ}$. As discussed earlier, the effective camber of the airfoil with GF increases causing earlier stall.

The decrease in $C_{L} / C_{D}$ ratio per every 10,000 decreases in Reynolds number for different ranges of Reynolds number is presented in Table 5 . This decrement seems to be nearly constant for the airfoil without and with GF at all Reynolds number ranges. However as the Reynolds number range decreases the lift/drag ratio is decreasing at a faster rate. When Reynolds number decreases from $3.0 \times 10^{5}$ to $1.5 \times$ $10^{5}$, the decrease in $C_{L} / C_{D}$ ratio per every 10,000 decreases 
TABLE 4: Percentage Increment in $C_{D}$ per every 10,000 decrease in Reynolds number for different ranges of Reynolds number.

\begin{tabular}{|c|c|c|c|c|c|}
\hline \multicolumn{6}{|c|}{ Reynolds number range } \\
\hline AoA, $\alpha$ & $\begin{array}{l}3.0 \times 10^{5} \\
\text { to } 1.5 \times 10^{5} \\
\end{array}$ & $\begin{array}{l}1.5 \times 10^{5} \\
\text { to } 1.0 \times 10^{5}\end{array}$ & $\begin{array}{l}1.0 \times 10^{5} \\
\text { to } 8.0 \times 10^{4} \\
\end{array}$ & $\begin{array}{c}8.0 \times 10^{4} \\
\text { to } 5.0 \times 10^{4} \\
\end{array}$ & $\begin{array}{c}5.0 \times 10^{4} \\
\text { to } 3.0 \times 10^{4}\end{array}$ \\
\hline \multicolumn{6}{|l|}{$4^{\circ}$} \\
\hline Without GF & 1.27 & 2.52 & 3.59 & 5.71 & 10.30 \\
\hline With GF & 0.73 & 1.51 & 2.18 & 3.45 & 6.40 \\
\hline \multicolumn{6}{|l|}{$8^{\circ}$} \\
\hline Without GF & 1.30 & 2.58 & 3.66 & 5.78 & 10.26 \\
\hline With GF & 0.83 & 1.62 & 2.31 & 3.56 & 6.25 \\
\hline \multicolumn{6}{|l|}{$12^{\circ}$} \\
\hline Without GF & 1.39 & 2.65 & 3.69 & 5.81 & 10.07 \\
\hline With GF & 1.03 & 1.91 & 2.62 & 3.94 & 6.60 \\
\hline
\end{tabular}

TABLE 5: Decrement in lift-to-drag ratio per every 10,000 decrease in Reynolds number for different ranges of Reynolds number.

\begin{tabular}{|c|c|c|c|c|c|}
\hline \multicolumn{6}{|c|}{ Reynolds number range } \\
\hline AoA, $\alpha$ & $\begin{array}{l}3.0 \times 10^{5} \\
\text { to } 1.5 \times 10^{5} \\
\end{array}$ & $\begin{array}{l}1.5 \times 10^{5} \\
\text { to } 1.0 \times 10^{5} \\
\end{array}$ & $\begin{array}{l}1.0 \times 10^{5} \\
\text { to } 8.0 \times 10^{4}\end{array}$ & $\begin{array}{c}8.0 \times 10^{4} \\
\text { to } 5.0 \times 10^{4}\end{array}$ & $\begin{array}{c}5.0 \times 10^{4} \\
\text { to } 3.0 \times 10^{4}\end{array}$ \\
\hline \multicolumn{6}{|l|}{$4^{\circ}$} \\
\hline Without GF & 0.262 & 0.454 & 0.595 & 0.793 & 1.161 \\
\hline With GF & 0.255 & 0.483 & 0.663 & 0.990 & 1.551 \\
\hline \multicolumn{6}{|l|}{$8^{\circ}$} \\
\hline Without GF & 0.386 & 0.641 & 0.824 & 1.095 & 1.552 \\
\hline With GF & 0.317 & 0.551 & 0.734 & 0.987 & 1.465 \\
\hline \multicolumn{6}{|l|}{$12^{\circ}$} \\
\hline Without GF & 0.375 & 0.572 & 0.698 & 0.910 & 1.256 \\
\hline With GF & 0.363 & 0.512 & 0.593 & 0.710 & 0.910 \\
\hline
\end{tabular}

in Reynolds number varies from 0.262 to 0.375 for the airfoil without GF as the angle of attack increases from $4^{\circ}$ to $12^{\circ}$. The corresponding values for the airfoil with GF are 0.255 and 0.363 . When Reynolds number decreases from $3.0 \times 10^{5}$ to $1.5 \times 10^{5}$, the corresponding values for the airfoil without and with GF are 1.161 and 1.256 and 1.551 and 0.910 , respectively.

3.2.4. Lift-to-Drag Ratio versus Lift Coefficient. The lift-todrag ratio as a function of lift coefficient for the airfoil without and with GF is presented in Figure 8. For the sake of clarity, the lift-to-drag ration is presented at four Reynolds numbers only $\left(\operatorname{Re}=3.0 \times 10^{4}, 8 \times 10^{4}, 1.5 \times 10^{5}\right.$ and $\left.3.0 \times 10^{5}\right)$. The liftto-drag ratio increases as Reynolds number increases for both the airfoils without and with GF. At lower lift coefficients, there is a drag penalty associated with GF. This drag penalty increases with Reynolds number. At higher lift coefficients, the lift-to-drag ratio increased. Hence the lift coefficient increases for a given lift-to-drag ratio. However at the highest lift coefficient, the lift-to-drag ratio is substantially reduced indicating large drag penalty as the airfoil approaches stall angle. This is true for the airfoil without GF and with GF at all Reynolds numbers.
3.2.5. Static Pressure Distribution. Static pressure distribution in terms of nondimensional coefficient $C_{P}$ is presented in Figure 9. For the sake of clarity, the static pressure distribution is presented for three Reynolds numbers only $(\mathrm{Re}=3.0 \times$ $10^{4}, 8 \times 10^{4}$ and $1.5 \times 10^{5}$ ) for the airfoil with and without Gurney flap at AoA $=12^{\circ}$, which is close to the stall angle. For the airfoil without Gurney flap, static pressure distributions are almost overlapping with each other for different Reynolds numbers. The effect of Reynolds number is clearly visible for the airfoil with Gurney flap. As the Reynolds number decreases, suction and pressure are continuously decreasing all along both the respective surfaces.

Maximum pressure build-up is almost the same for the airfoils with and without GF. Value of maximum $C_{P}$ for variation in Reynolds number from $\operatorname{Re}=3.0 \times 10^{4}$ to $3.0 \times$ $10^{5}$ varies from 1.11 to 1.02 for the airfoil without GF and from 1.09 to 1.02 for the airfoil with GF. The maximum suction decreases as Reynolds number decreases. However, the change is very small for the airfoil without GF. Maximum suction varies from -3.4 to -4.6 for $\mathrm{Re}=3.0 \times 10^{4}$ to $3.0 \times 10^{5}$ for the airfoil without GF, whereas, for the same variation in Reynolds number, $C_{P}$ values vary from -4.5 to -6.8 for the airfoil with GF. On the pressure surface, the static pressure 


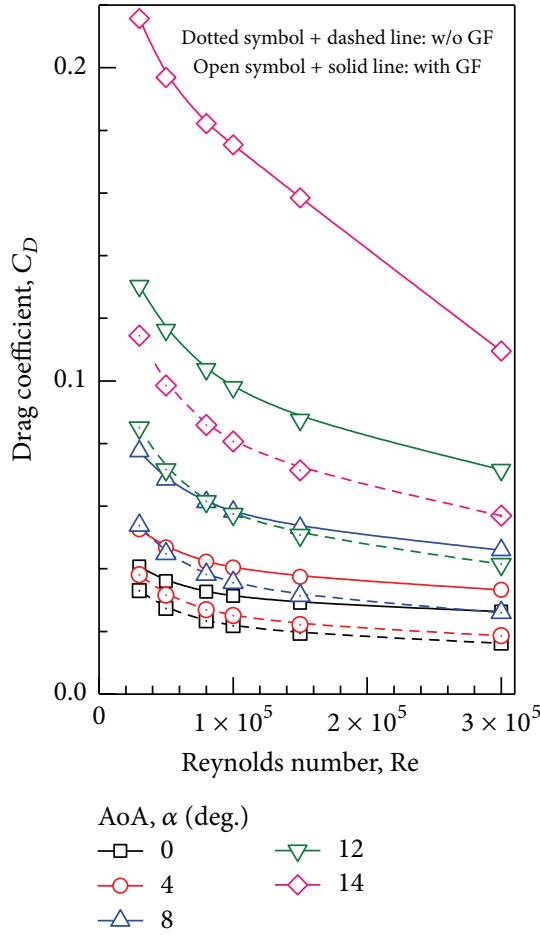

Figure 6: Variation of $C_{D}$ with Reynolds number for the airfoil without and with GF.

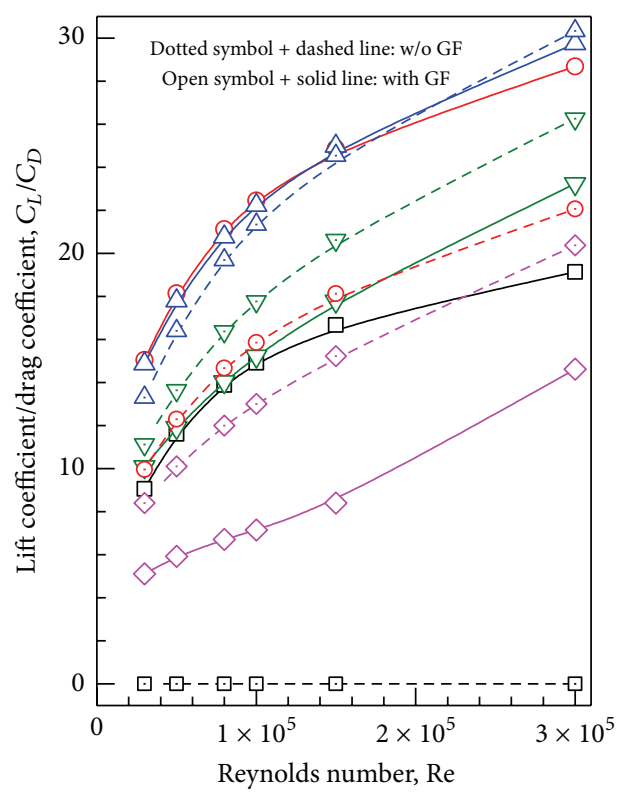

$\begin{array}{ll}\text { AoA, } \alpha \text { (deg.) } & \\ -\square-0 & -\nabla-12 \\ -\bigcirc-4 & \checkmark-14 \\ -\triangle 8 & \end{array}$

FIGURE 7: Variation of lift-to-drag ratio with Reynolds number for the airfoil without and with GF.

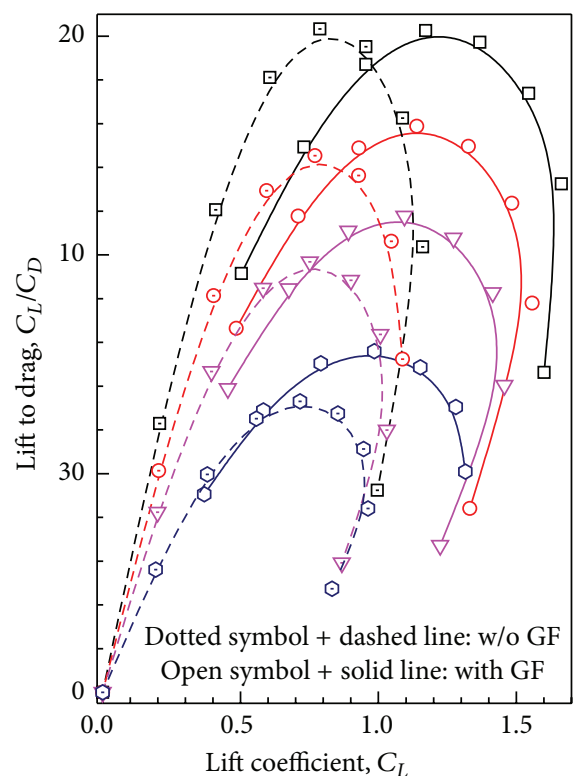

$$
\begin{array}{cc}
\operatorname{Re} & \mathrm{Re} \\
-\square-3.0 \times 10^{5} & -\nabla^{-} 8 \times 10^{4} \\
-\bigcirc-1.5 \times 10^{5} & -\square-3 \times 10^{4}
\end{array}
$$

FIGURE 8: Variation of lift-to-drag ratio with lift coefficient at different Reynolds numbers for the airfoil without and with GF.

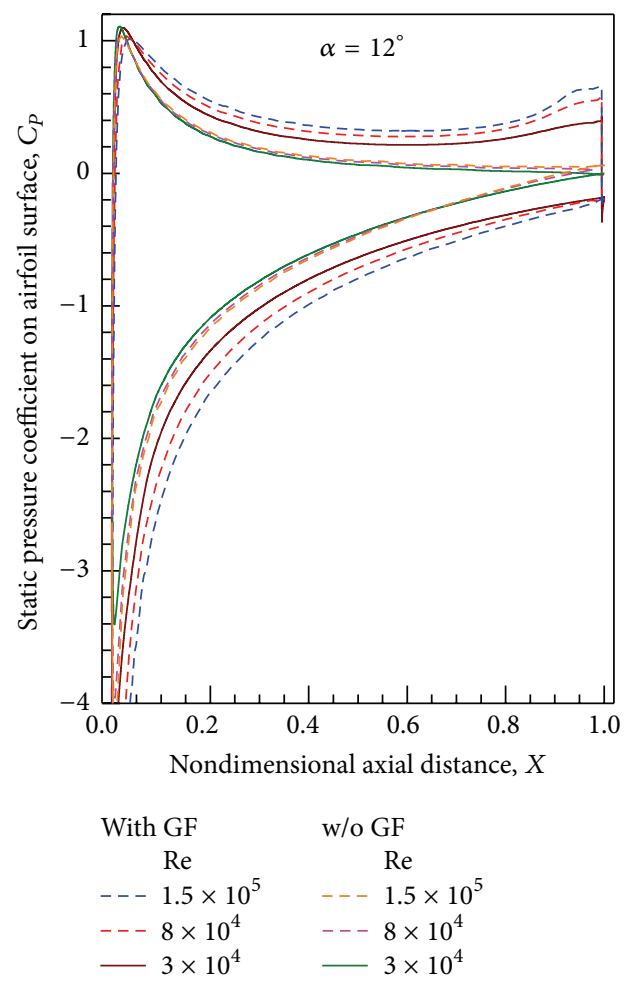

FIGURE 9: Static pressure distributions for different Reynolds numbers at $\mathrm{AoA}=12^{\circ}$ for the airfoil without and with GF. 
With GF

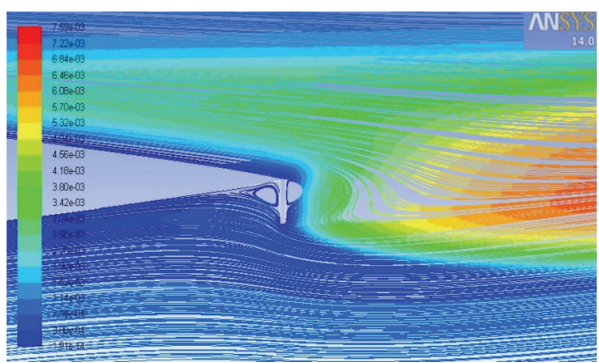

$\operatorname{Re}=3.0 \times 10^{5}$

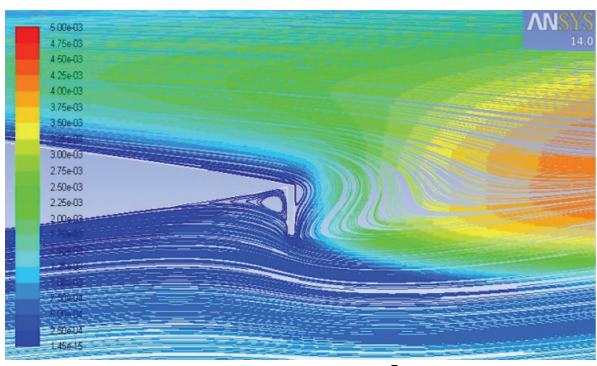

$\mathrm{Re}=1.5 \times 10^{5}$

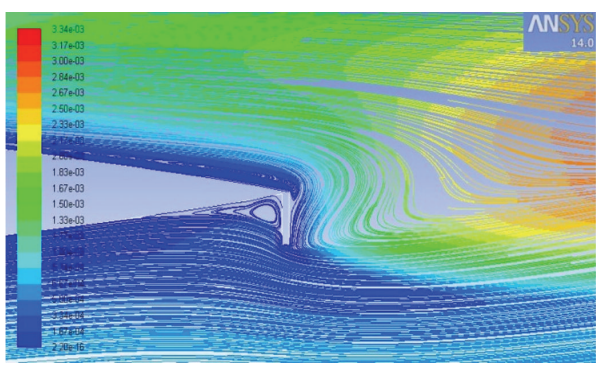

$\mathrm{Re}=8.0 \times 10^{4}$

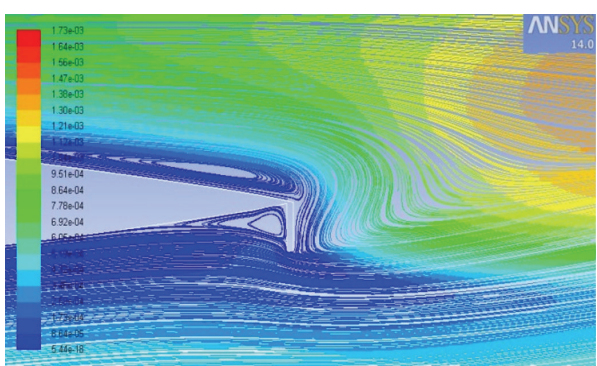

$\operatorname{Re}=3.0 \times 10^{4}$
Without GF

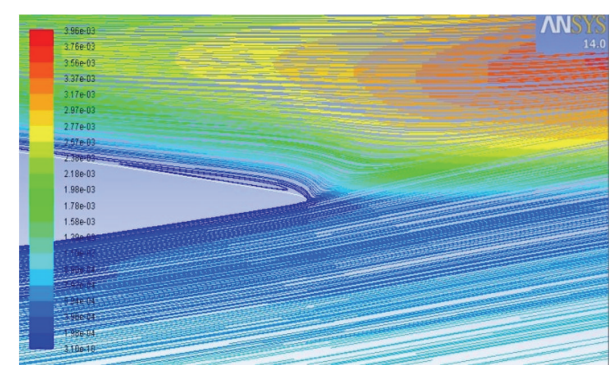

$\operatorname{Re}=3.0 \times 10^{5}$

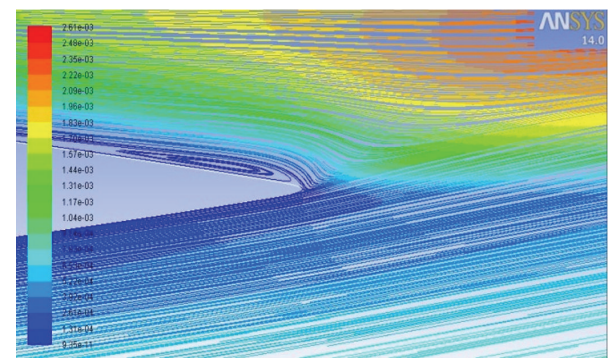

$\operatorname{Re}=1.5 \times 10^{5}$

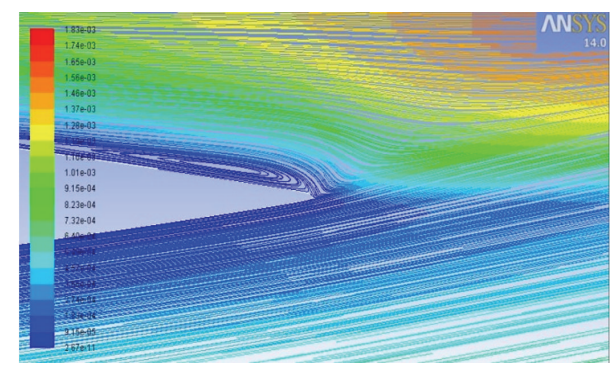

$\operatorname{Re}=8.0 \times 10^{4}$

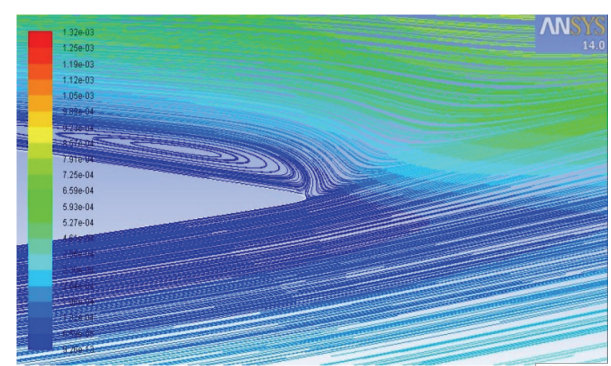

$\mathrm{Re}=3.0 \times 10^{4}$

FIGURE 10: Pathlines and turbulent viscosity for different Reynolds numbers at $\mathrm{AoA}=12^{\circ}$ of the airfoil with and without Gurney flap.

increases for the airfoil with GF, while, on the suction surface, the static pressure decreases. This occurs all along the surface resulting in substantial increase in lift coefficient for the airfoil with GF at all angles of attack and at all Reynolds numbers. Adverse pressure gradient occurs on the pressure surface near the trailing edge of the airfoil with GF. This adverse pressure gradient decreases with Reynolds number. Such adverse pressure gradients were experimentally observed by previous researchers [20]. A recirculating vortex that occurs just upstream of Gurney flap may be the reason for this adverse pressure gradient [2]. Static pressure coefficients are reduced near the Gurney flap as Reynolds number decreases. Unlike the sudden rise in pressure just before Gurney flap on pressure surface at high Reynolds numbers, rate of pressure build-up is not rapid at low Reynolds numbers.

3.2.6. Pathlines. Pathlines are highly affected by the variation in the Reynolds number and the formation of vortices experience drastic change. Pathlines superimposed with the contours of turbulent viscosity at $\mathrm{AoA}=12^{\circ}$ for the airfoil without and with GF at four different Reynolds numbers presented in Figure 10. Unlike at very high Reynolds number (of the order of $\operatorname{Re}=10^{6}$ ), only one vortex is completely formed in the wake of Gurney flap at $\mathrm{Re}=3.0 \times 10^{5}$ instead of two vortices. As the Reynolds number further decreases, the only vortex in wake also start to disappear. 
A laminar separation bubble starts its formation. As the Reynolds number is decreased, the flow loses its ability to make transition into turbulent flow in the attached boundary layer, hence forming a laminar separation bubble. Below $\operatorname{Re}=1.0 \times 10^{5}$, no vortex is present behind the Gurney flap. The increased pressure on pressure surface is only due to vortex ahead of the Gurney flap which might explain absence of sudden pressure increase near the Gurney flap as shown in static pressure distribution. This laminar separation bubble increases the effective thickness of the airfoil, thereby increasing the pressure drag over the region, which explains the increase in drag at low Reynolds number and degraded performance of the airfoil at low Reynolds numbers. For each Reynolds number, flow is turned towards the Gurney flap whereas, due to absence of any suction, the flow leaves at the airfoil at higher angle without Gurney flap. However the turning of flow towards GF is reduced at lower Reynolds numbers.

\section{Conclusions}

A computational investigation on the effects of Reynolds number on the aerodynamics of NACA0012 airfoil without and with Gurney flap of height of 3\% airfoil chord has been carried out. ANSYS FLUENT commercial CFD code with one-equation Spalart-Allmaras turbulence model is used for the six Reynolds numbers varying from $3.0 \times 10^{5}$ to $3.0 \times 10^{4}$. From this investigation, the following major conclusions are drawn.

(1) Reynolds number plays a very major role in the airfoil aerodynamics for the NACA0012 airfoil without and with Gurney flap. Lift decreases and drag increases when Reynolds number is decreased.

(2) For the airfoil with GF, Reynolds number has adverse effects on lift coefficient, while drag coefficient of the airfoil with GF has some beneficial effects compared to the airfoil without GF.

(3) For high Reynolds number above critical range, decrease in $C_{L}$ and increase in $C_{D}$ are negligible.

(4) As the Reynolds number is decreased below the critical Reynolds number range, $C_{L}$ decreases and $C_{D}$ increases at very steep rate and the performance degrades rapidly.

(5) For lower Reynolds numbers, the two vortices behind the Gurney flap vanish. The Gurney flap seems to increase the effective camber of the airfoil, causing negative zero lift angle and reduced stall angle.

\section{Nomenclature}

AoA, $\alpha$ : Angle of attack (deg.)

$C_{D}: \quad$ Drag coefficient $=2 L / \rho U^{2}$

Ch: Airfoil chord (m)

$C_{L}: \quad$ Lift coefficient $=2 D / \rho U^{2}$

$C_{P}: \quad$ Static pressure coefficient $=$ $2\left(p_{s}-p_{s 2}\right) / \rho U^{2}$

$D: \quad$ Drag force $(\mathrm{N})$ $h$ : Optimum Gurney flap (m)

$H$ : Gurney flap height as percentage of chord

$L: \quad$ Lift force $(\mathrm{N})$

$p_{s}:$ Static pressure $(\mathrm{Pa})$

Re: Reynolds number $=U \mathrm{Ch} / \nu$

$U$ : Freestream velocity $(\mathrm{m} / \mathrm{s})$

$\varepsilon$ : Rate of dissipation of turbulent kinetic energy $\left(\mathrm{m}^{2} / \mathrm{s}^{3}\right)$

$\kappa:$ Turbulent kinetic energy $\left(\mathrm{m}^{2} / \mathrm{s}^{2}\right)$

$\rho:$ Air density $\left(\mathrm{kgm} / \mathrm{m}^{3}\right)$

$v$ : Kinematic viscosity $\left(\mathrm{m}^{2} / \mathrm{s}\right)$.

Subscripts

2: Exit.

\section{Abbreviations}

CFD: Computational fluid dynamics

GF: $\quad$ Gurney flap

$\kappa-\varepsilon$ RNG: $\kappa-\varepsilon$ RNG turbulence model

RANS: Reynolds averaged Navier Stokes equations

SA: $\quad$ Spalart-Allmaras turbulence model.

\section{Conflict of Interests}

The authors declare that there is no conflict of interests regarding the publication of this paper.

\section{References}

[1] R. W. Prouty, Helicopter Aerodynamics, vol. 2, Philips Publishing, 1985.

[2] R. H. Liebeck, "Design of subsonic aerofoils for high lift," Journal of Aircraft, vol. 1, no. 9, pp. 547-561, 1978.

[3] Y. C. Li, J. J. Wang, and P. F. Zhang, "Effects of Gurney flaps on a NACA0012 airfoil," Flow, Turbulence and Combustion, vol. 68, no. 1, pp. 27-39, 2002.

[4] J. J. Wang, Y. C. Li, and K.-S. Choi, "Gurney flap-Lift enhancement, mechanisms and applications," Progress in Aerospace Sciences, vol. 44, no. 1, pp. 22-47, 2008.

[5] T. J. Mueller and S. M. Batill, "Experimental studies of separation on a two-dimensional the airfoil at low Reynolds numbers," AIAA journal, vol. 20, no. 4, pp. 457-463, 1982.

[6] M. S. Selig, J. F. Donovan, and D. B. Fraser, Airfoils at Low Speeds, Soartech 8, Soartech Publications, Virginia Beach, Va, USA, 1989.

[7] L. Brown and A. Filippone, "Aerofoil at low speeds with Gurney flaps," The Aeronautical Journal, vol. 107, no. 1075, pp. 539-546, 2003.

[8] L. W. Traub and G. Agarwal, "Aerodynamic characteristics of a gurney/jet flap at low reynolds numbers," Journal of Aircraft, vol. 45, no. 2, pp. 424-429, 2008.

[9] T. J. Mueller, "Aerodynamic measurements at low Reynolds numbers for fixed wing micro-air vehicles," in Proceedings of the Special Course on Development and Operations of UAVs for Military and Civil Applications, Rhode-Saint-Genèse, Belgium, September 1999. 
[10] G. K. Ananda, P. Sukumar, and M. S. Selig, "Low-to-moderate aspect ratio wings tested at low Reynolds numbers," AIAA Paper 3026, 2012.

[11] M. Suresh and N. Sitaram, "Gurney flap applications for aerodynamic flow control," in Proceedings of the 9th International Conference on Mechanical Engineering, Paper no. ICME 11-FL40, Dhaka, Bangladesh, December 2011.

[12] K. Sriram, S. Jain, and N. Sitaram, "Grid and turbulence model based exhaustive analysis of NACA 0012 airfoil," Journal of Advanced Research in Applied Mechanics and Computational Fluid Dynamics, vol. 1, no. 1, pp. 13-18, 2014.

[13] S. Jain, N. Sitaram, and S. Krishnaswamy, "Computational investigations on the effects of gurney flap on airfoil aerodynamics," International Scholarly Research Notices, vol. 2015, Article ID 402358, 11 pages, 2015.

[14] Ansys, ANSYS Fluent Theory Guide, Ansys, Canonsburg, Pa, USA, 2011.

[15] J. N. Masoud, S. Habibollah, and A. Mekanik, "Numerical investigation of aerodynamic characteristics of NACA 23018 airfoil with a gurney flap," International Journal of Mechanical Engineering and Robotics Research, vol. 1, no. 3, pp. 341-349, 2012.

[16] B. M. Arko and M. McQuilling, "Computational study of highlift low-pressure turbine cascade aerodynamics at low reynolds number," Journal of Propulsion and Power, vol. 29, no. 2, pp. 446-459, 2013.

[17] K. Abe, T. Kondoh, and Y. Nagano, "A new turbulence model for predicting fluid flow and heat transfer in separating and reattaching flows-I. Flow field calculations," International Journal of Heat and Mass Transfer, vol. 37, no. 1, pp. 139-151, 1994.

[18] K. Abe, T. Kondoh, and Y. Nagano, "A new turbulence model for predicting fluid flow and heat transfer in separating and reattaching flows-II. Thermal field calculations," International Journal of Heat and Mass Transfer, vol. 38, no. 8, pp. 1467-1481, 1995.

[19] M. Kato and B. E. Launder, "The modeling of turbulent flow around stationary and vibrating square cylinders," in Proceedings of the 9th Symposium on Turbulent Shear Flows, pp. 10.4.110.4.6, Kyoto University, Kyoto, Japan, August 1993.

[20] R. Myose, I. Heron, and M. Papadakis, "The post-stall effect of gurney flaps on a NACA-0011 airfoil," in Proceedings of the Aerospace Atlantic Conference, SAE Paper 961316, Dayton, Ohio, USA, May 1996. 

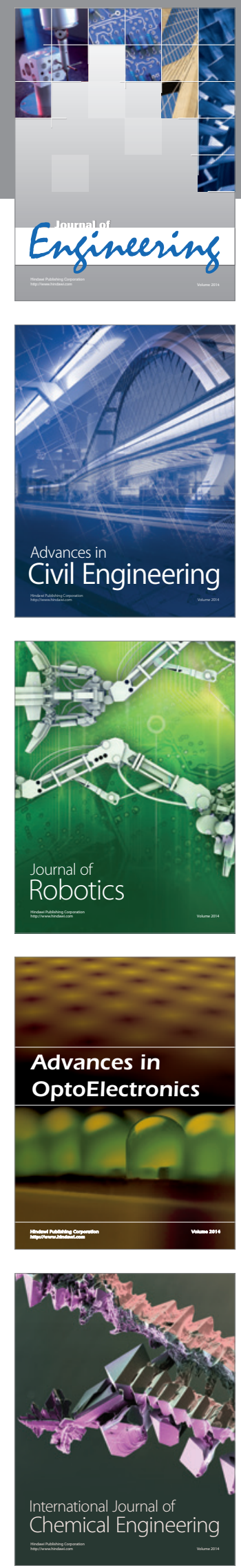

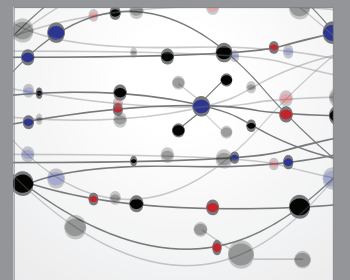

The Scientific World Journal
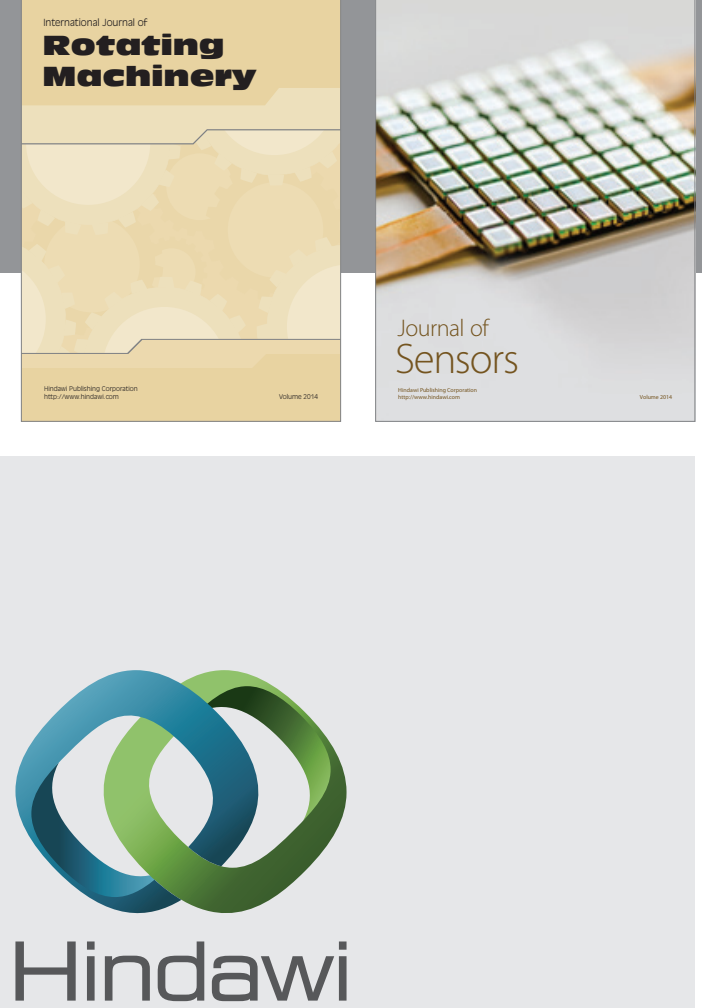

Submit your manuscripts at http://www.hindawi.com
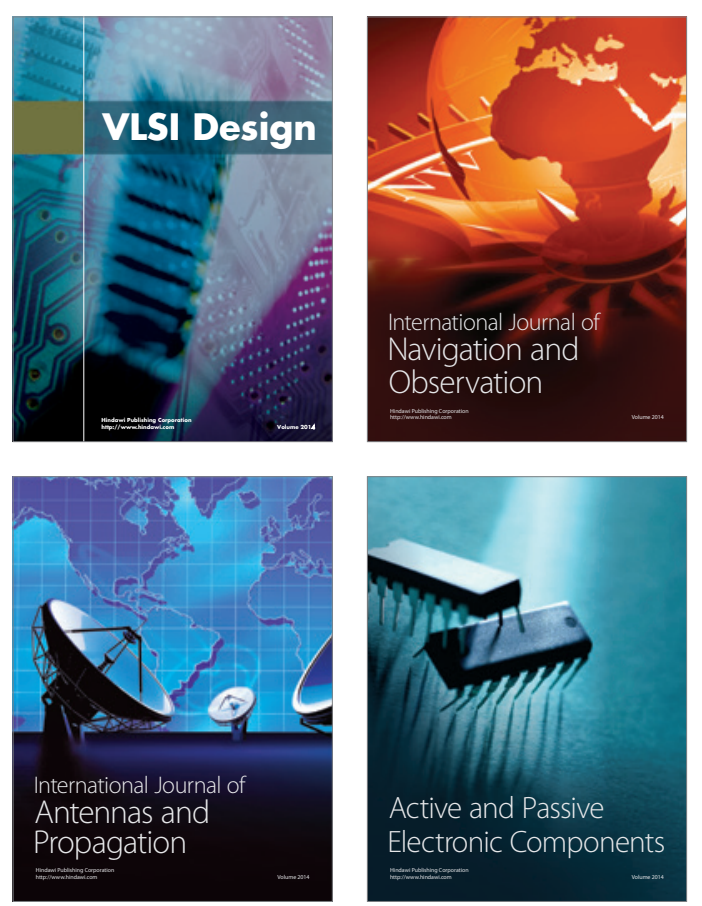
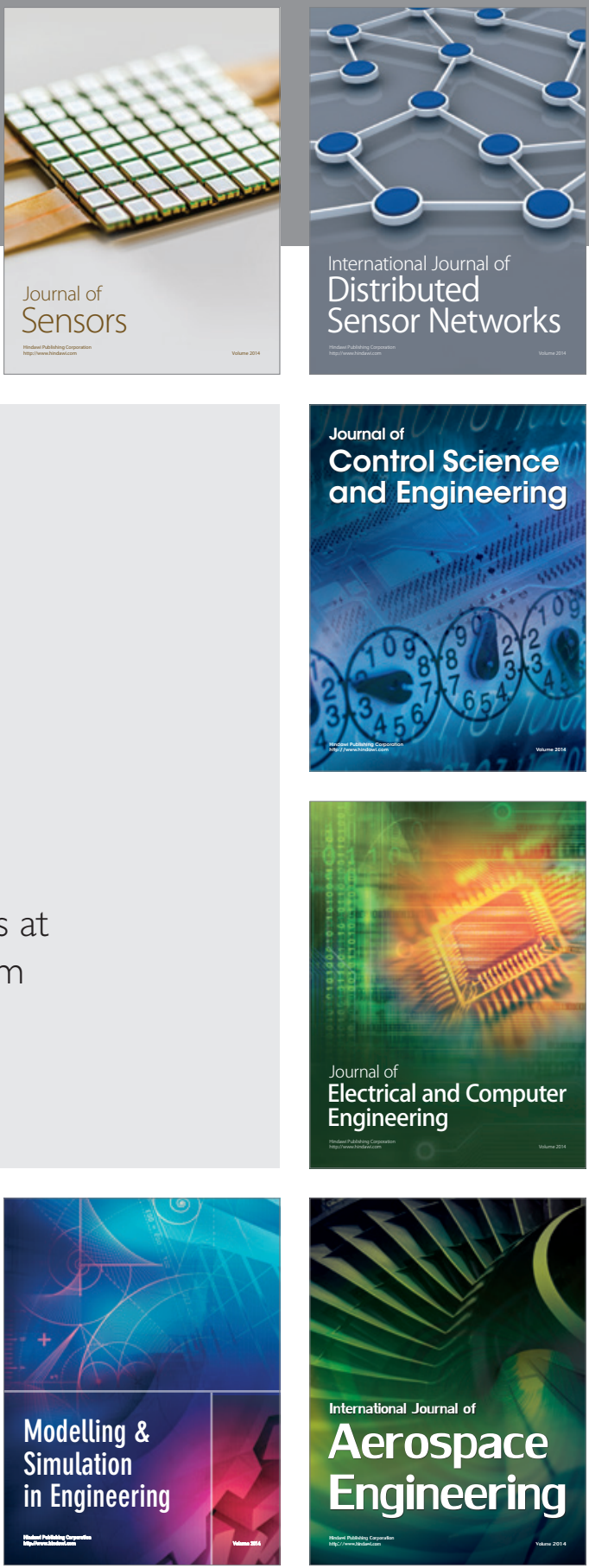

Journal of

Control Science

and Engineering
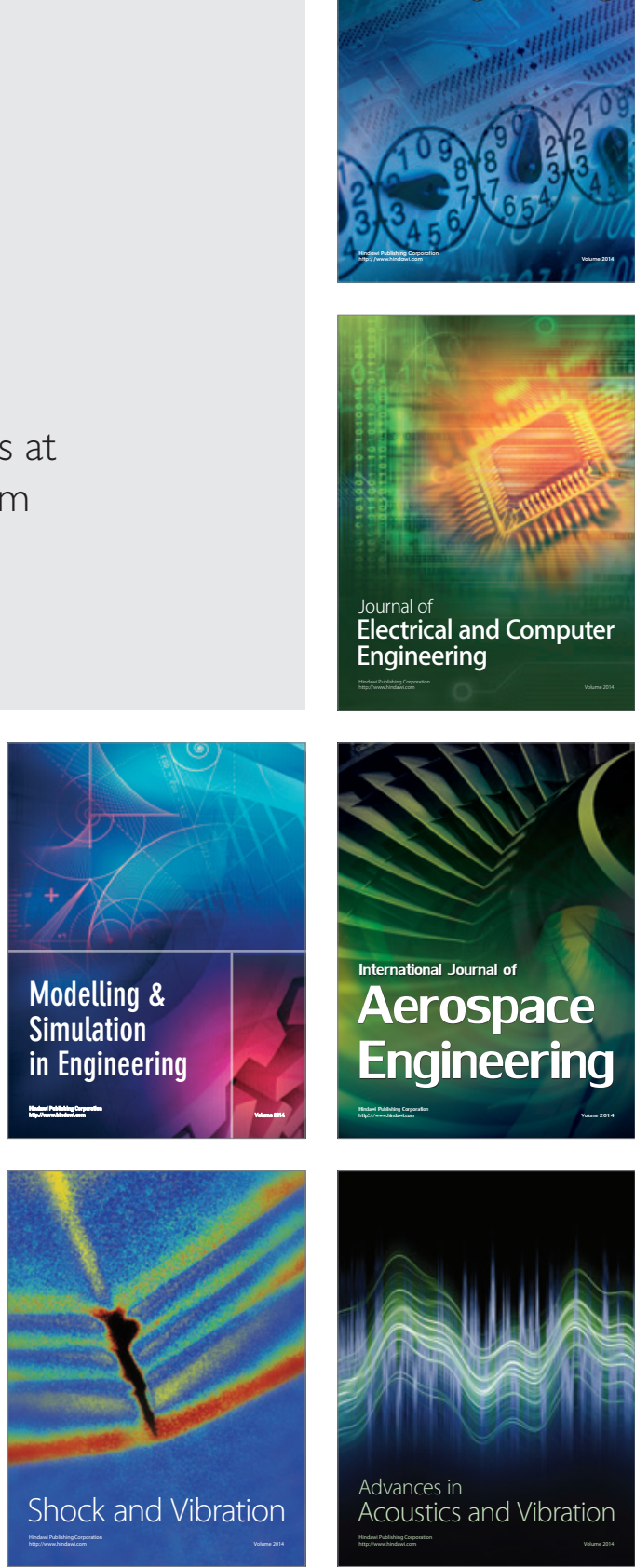